\title{
NFC2BLE Communications' Bridge: From Flash to Continuous Monitoring of Biological Parameters
}

\author{
Jose Maria Vicente ${ }^{1,}$, Ernesto Avila-Navarro², Alberto Rodriguez-Martinez ${ }^{3}$, \\ Miguel Angel de la Casa Lillo ${ }^{1}$, Jose Maria Sabater-Navarro ${ }^{1}$ \\ ${ }^{1}$ Bioengineering Institute, Miguel Hernandez University of Elche, \\ ${ }^{2}$ Department of Material Science, Optics and Electronic Tec., Miguel Hernandez University of Elche, \\ ${ }^{3}$ Department of Communications Engineering, Miguel Hernandez University of Elche, \\ Avinguda de la Universitat d'Elx, 03202 Elche, Alicante, Spain \\ jose.vicentes@umh.es
}

\begin{abstract}
${ }^{1}$ Abstract-The devices for monitoring biological variables have become a hot topic in the last years. An important aspect in the design of these wearable devices is the continuous communication, as well as the associated energy consumption. As a result, the concept of flash monitoring has been recently coined. This implies that the reading of the variables is not made in a continuous manner, but the values are stored on the sensor electronics for a while and then retrieved altogether to the reader. This concept reduces the energy consumption since there is no continuous communication, and this feature is, therefore, beneficial in certain contexts. However, the communication is sometimes required to be continuous, so that solutions based on continuous communication or alarms can be implemented. This work shows a technological development that allows converting a biological variable reading system based on flash monitoring into a continuous one. The details of the electronic development of the components are shown, and the software structure integrated in the microcontroller is discussed. The system devoted to the management and remote acquisition of the data is shown as well. As an example, the performance of the system is shown in two different contexts: with a flash biopatch for the body temperature monitoring designed in the laboratory and with a commercially available flash interstitial glucose monitoring system.
\end{abstract}

Index Terms-Biomedical telemetry; Electronic circuits; Bluetooth; Near-field communication.

\section{INTRODUCTION}

An important aspect in the design of wearable devices is the continuous communication, as well as the associated energy consumption.

There are many devices for monitoring physiological parameters that use Bluetooth Low Energy (BLE) technology to transmit information to a central device for managing this data, i.e. from chest bands for the measurement of heart rate used in sports activities or wristbands for acquiring body activity until the appearance of new wearables with the integration of many sensors. For

Manuscript received 2 October, 2019; accepted 12 January, 2020.

This research was funded by a grant (No. DPI2016-80391-C3-2-R [AEI/FEDER, UE]) from the Agencia Estatal de Investigación (AEI) and from EU through the Fondo Europeo de Desarrollo Regional - FEDER "A way to build Europe". example, since Apple Watch Series 4 [1], it incorporates an electrical sensor, in addition to the optical, for the measurement of heart rate and that allows measuring the electrocardiogram signal (ECG). There are also devices that allow the measurement of the electrodermal activity (EDA), such as the Embrace of Empatica [2] used for epilepsy management. Even the concept of smart clothes starts to appear [3]. The incorporation of textile electronics for monitoring is a fact. An example of this technology is Hexoskin [4], which integrates electrodes on the t-shirts allowing a direct and comfortable contact with the user's skin.

The continuous transmission of data is an advantage of BLE communication. However, it has disadvantages also. This type of devices has a high-power consumption that makes the autonomy not the desired one. In addition, most of these devices need a receiving device (reader) to get the data. This must be found near the user at all times too.

One commonly used option to reduce energy consumption is to implement Near-Field Communications (NFC) based solutions. Within the NFC devices, there are those so-called "biopatchs". These products are destined to monitoring physiological signals with NFC communication integrated. These devices allow controlling some physical parameters of the user in a flash mode storing a certain amount of information on the tag electronics and retrieving this data to the reader when the NFC communication is established. The user needs to place the reader near to scan the tag on demand. There are several commercial biopatchs in market, such as FreeStyle Libre from Abbot Laboratories [5] to control glucose, TempTraq from Blue Spark Technologies [6] to control temperature or femSense from SteadySense GmbH [7] to detect ovulation. Moreover, there are many research prototypes to measure all kinds of variables [8], [9].

The flash scheme implies that the reading of the variables is not made in a continuous manner, but the values are stored for a while and then retrieved altogether. However, the communication is sometimes required to be continuous, so that the solutions based on continuous communication or alarms can be implemented. This work shows a 
technological development that builds an NFC-to-BLE electronic bridge that allows converting a variable reading system based on flash monitoring into a continuous one by demand.

The paper is organized as follows. Section II details the device design and presents the design parameters of BLE and NFC antennas. Section III shows the software development for implementing the communications' bridge. Section IV gives information of how to implement an Android app for reading the parameters. Finally, Section V shows two implementations for the applications of reading a temperature biopatch and a commercial flash interstitial glucose monitoring system.

\section{DEVICE DESCRIPTION}

This section presents the design of the electronic device that acts as a bridge between the biopatch sensor and a smartphone with BLE. There are many important aspects, such as size, comfort or allocation, that must be consider when designing a device that interacts with the user and that must also be positioned on the user's body. The grouping of different parts forms the physical design of the proposed device. Each of these parts have been developed trying to provide the required characteristics for its use, such as materials of manufacture, geometry, location or selection of components.

The device has overall dimensions of $80 \times 70$ millimetres visibly divided into two parts, one flexible to facilitate comfort to the user and other part rigid to robust the electronics welding of the components, and its geometry is rectangular. Figure 1 shows the bottom and upper views of the device.

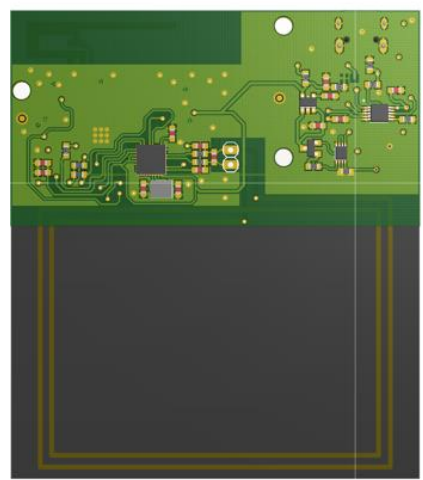

(a)

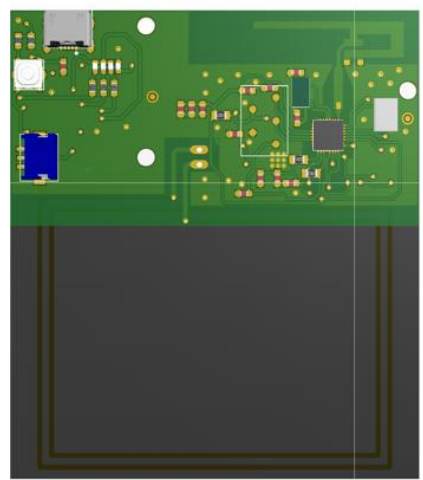

(b)
Fig. 1. (a) Bottom and (b) Upper views of the device.

\section{A. Flexible Electronics}

On the one hand, there is a flexible part with 84 microns of thickness. It is formed by an internal copper layer of 17 microns and covered with polyamide. In this part, the NFC antenna is located. The choice of this material for the manufacture of this part of the device brings comfort to the user. The flexible material adapts to the curvature of the user's body avoiding generating discomfort or even possible injuries during sleep periods. The total dimensions of the flexible part are 70x43 millimetres, i.e. $53.75 \%$ of the surface of the device. This part should be positioned over the flash biopatch that the user has placed.

The geometry of the NFC antenna is rectangular, with dimensions of $60 \times 45$ millimetres. It consists of 9 rectangular copper segments with $0.8 \mathrm{~mm}$ wide and 17 microns of thickness, which form 2 complete turns. The separation of the centres of segments between turns is 2 millimetres. Figure 2 shows the layout of the NFC antenna designed for the device.

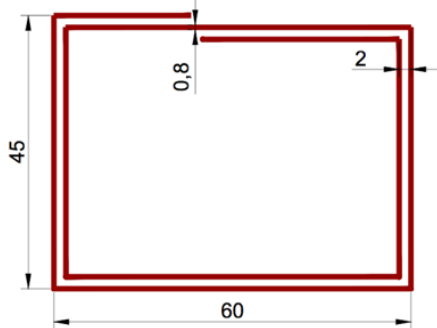

Fig. 2. NFC antenna layout.

Figure 3 plots that the real inductance of the antenna measured with a VNA (Vector Network Analyzer) is $613.3 \mathrm{nH}$ (at $13.56 \mathrm{MHz}$ ). With the frequency measurements taken from the antenna, the system is adapted for operation in the NFC standard at $13.56 \mathrm{MHz}$ according to the manufacturer of the NFC transponder used in the device.

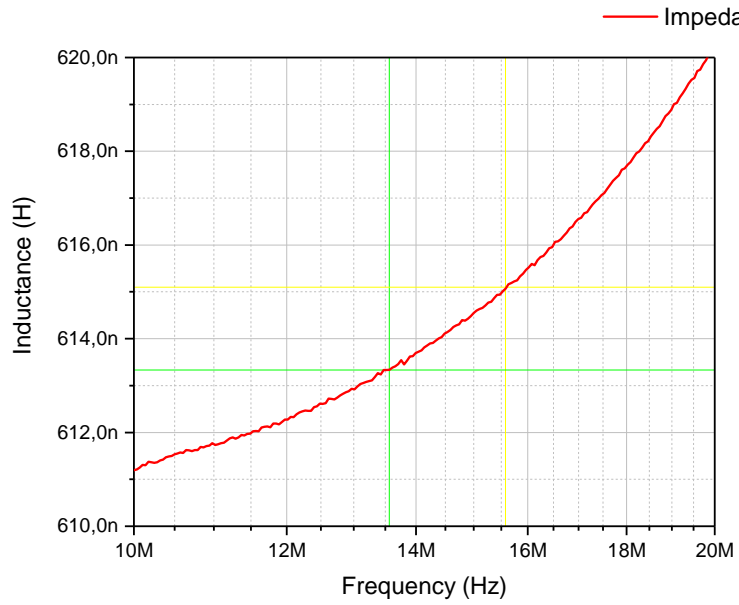

Fig. 3. Inductance of the NFC antenna.

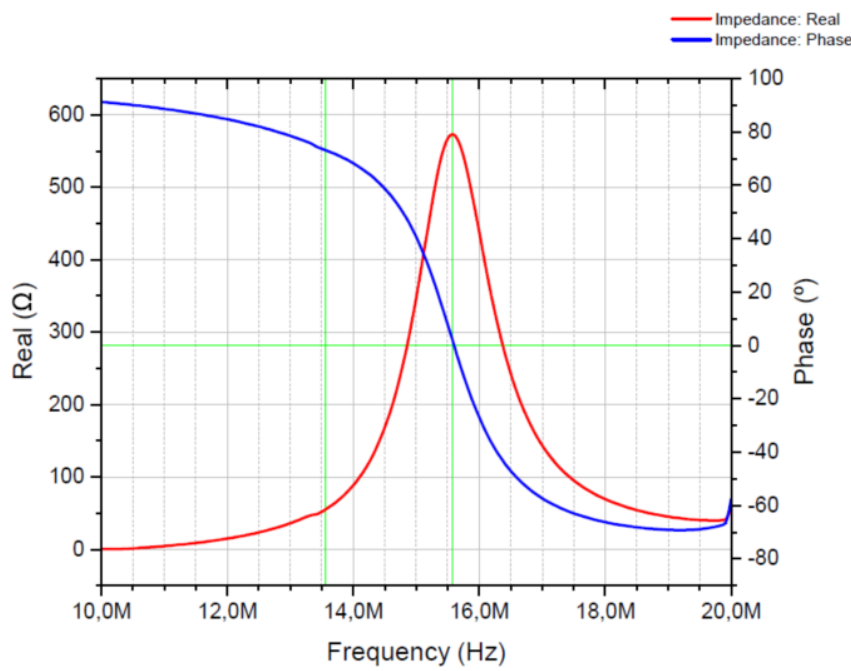

Fig. 4. Resonance frequency of the NFC system. Red line is real value of impedance $(\Omega)$. Blue line is impedance phase $\left({ }^{\circ}\right)$.

The adapted circuit is designed, so that its operating range is about $13.56 \mathrm{MHz}$. Despite this, the resonance frequency is greater than $13.56 \mathrm{MHz}$ due to anti-collision reasons. The resonance frequency is usually set to be between $1 \mathrm{MHz}$ and 
$5 \mathrm{MHz}$ higher to maintain the highest performance in case two or more transponders are in the area of the reading device. The reason for this is the fact that two transponders have a frequency smaller than a single transponder. Thus, in Fig. 4, it is observed that the resonance frequency of the system is $15.58 \mathrm{MHz}$, also measured with the VNA. The Quality - factor of the antenna is 10.5 with a bandwidth of 1.4 MHz. The system has a maximum communication distance of $10 \mathrm{~cm}$ (measured empirically).

\section{B. Rigid Part Electronics}

On the other hand, there is a rigid part that includes the power management of the device and the antenna for Bluetooth communication. This part of the device has a thickness of 0.871 millimetres distributed in 2 external copper layers of 35 microns, 2 internal copper layers of 17 microns, 1 dielectric section with polyamide (67 microns), and another dielectric section of FR4 (700 microns). The FR4 provides the rigidity necessary to avoid a variation in the impedance of the antenna for a good Bluetooth communication. The corresponding surface are the remaining 70x37 millimetres of the device. The geometry chosen for the Bluetooth antenna is an inverted F-type antenna. The choice of this type of antenna is due to its good performance and its contained size. The adopted solution tries to use the minimum number of physical components. It has been decided to adapt the inverted F-type antenna, so that it does not require additional passive components.

In Figure 5, the parameter S11 of the Bluetooth antenna is plotted vs frequency. For the operating frequency of the Bluetooth standard $(2.45 \mathrm{GHz})$, the return loss is $13.815 \mathrm{~dB}$, which corresponds to the highest radiation point of the antenna. The antenna has a gain of $1.26 \mathrm{~dB}$, its directivity is $2.4 \mathrm{~dB}$, and it has a radiation efficiency of $76.75 \%$. The antenna is designed and adapted to radiate in all directions. Figure 6 shows the radiation pattern of the antenna in planes $\mathrm{H}$ and $\mathrm{E}$. It can be seen how there is hardly any difference in the magnitude between both.

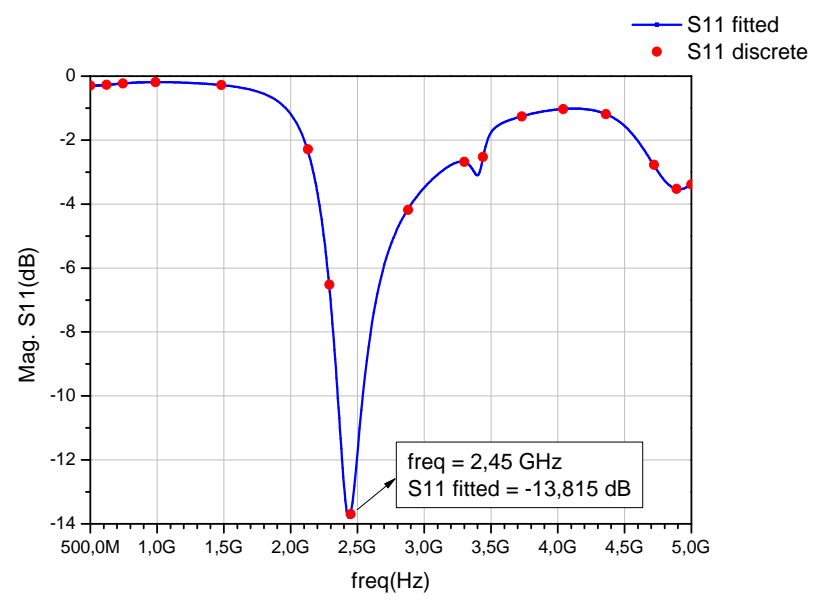

Fig. 5. S11 parameter magnitude of the Bluetooth antenna.

For the design of power management, three main aspects have been considered. First, get a reasonable autonomy since it is a portable device. It is also important that the device has easy interfaces for battery charging and use device. In addition, the system must be integrated into a reduced space in order not to increase the dimensions of the device excessively.

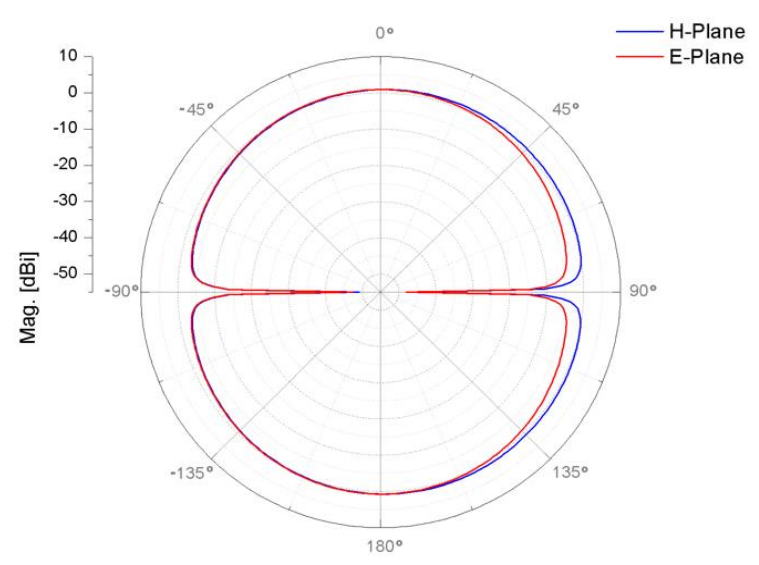

Fig. 6. Radiation pattern of the Bluetooth antenna in $\mathrm{H}$ and $\mathrm{E}$ planes.

For this, a $260 \mathrm{mAh}$ battery with only $35 \times 12 \times 6$ millimetres has been selected. The charging interface is a microUSB connector, which allows using a standard charger for smartphones making it easier to charge the device for the user.

\section{SOFTWARE IMPLEMENTATION}

The software loaded to the microcontrollers will allow managing the communications. A Texas Instrument CC2640 controller and a CR95HF RF transceiver have been selected to manage the device. The system is able to run an application that reads the memory of the NFC tag, preprocess the data and send it to the central device of the BLE pair. The sample period, management of the memory and pre-processing operations can be configured by programming.

Figures 7 and 8 show the flowcharts implemented on the TI CC2640 controller for the NFC and BLE stacks. As the Fig. 7 illustrates, after the initialization process, a timer manages the NFC readings and the data are stored in memory. The BLE stack is a commonly used implementation that notifies any new information obtained by the NFC readings. The pre-processing of the NFC readings is implemented in the BLE flow (Fig. 8).

\section{REMOTE APP}

To control the device, an Android app has been developed. It allows to manage alarms and to keep track of the parameter values taken by the sensor, and to compute statistics of the values. This application also acts as a link between the device and a database in the cloud. It allows the user to have stored the history of measured values over time and access them remotely through the internet from any place.

The application has been developed, so that it has a comfortable and simple use by users (user-friendly). It has a very easy to understand interface. It provides a central marker, where the last values measured by the sensor are displayed. To give a glimpse of the connection status, two labels display the state of the connection and the last read status. Three additional buttons are located on the interface. The left button allows forcing a manual measurement on the sensor. The central button, green, will turn red to indicate that the device is low battery. 


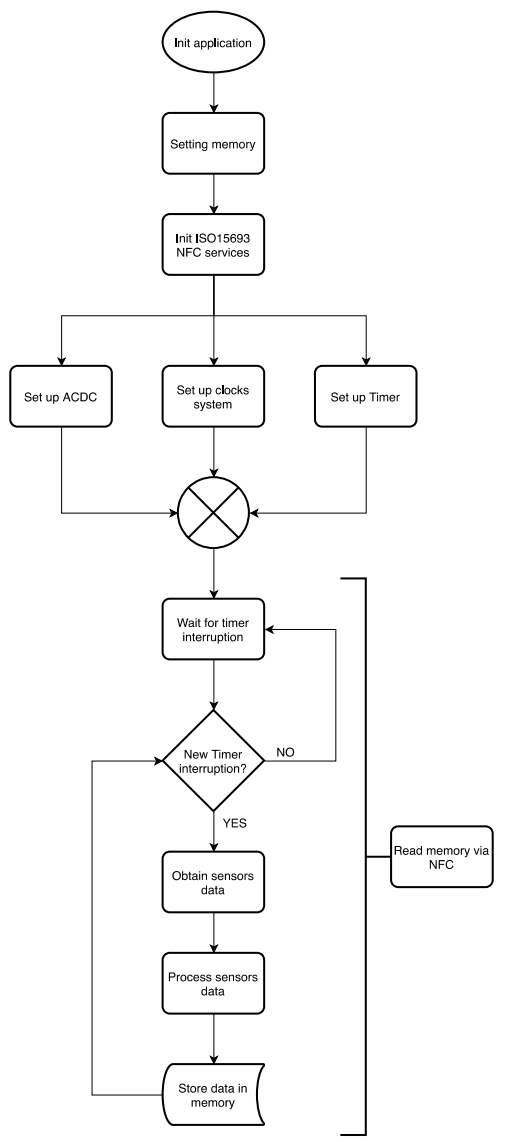

Fig. 7. Flowchart of the NFC stack.

Finally, the right button will take a snapshot of the temporal data graph and store the pic in the smartphone's gallery. This temporal data graph is located at the bottom of the interface. Figure 9 shows an image of the application interface when reading a glucose signal.

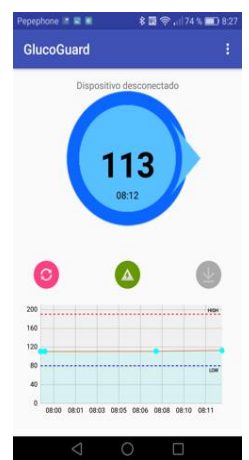

Fig. 9. Main interface of the Android app.

In addition to this main interface, there is a settings tab, in which a series of features and functions of the application that allow the user to take full advantage of the device can be configured. Some of these features are:

- To establish maximum and minimum limits for the read value;

- Sound alarm for values out of the limits;

- GSM call for values out of the limits;

- Alarm for lost readings. The device performs sensor automatic measurements every 5 minutes. If the application losses two consecutive measurements, it will alert the user to check the position just in case it has been displaced with respect to the sensor. This feature is very

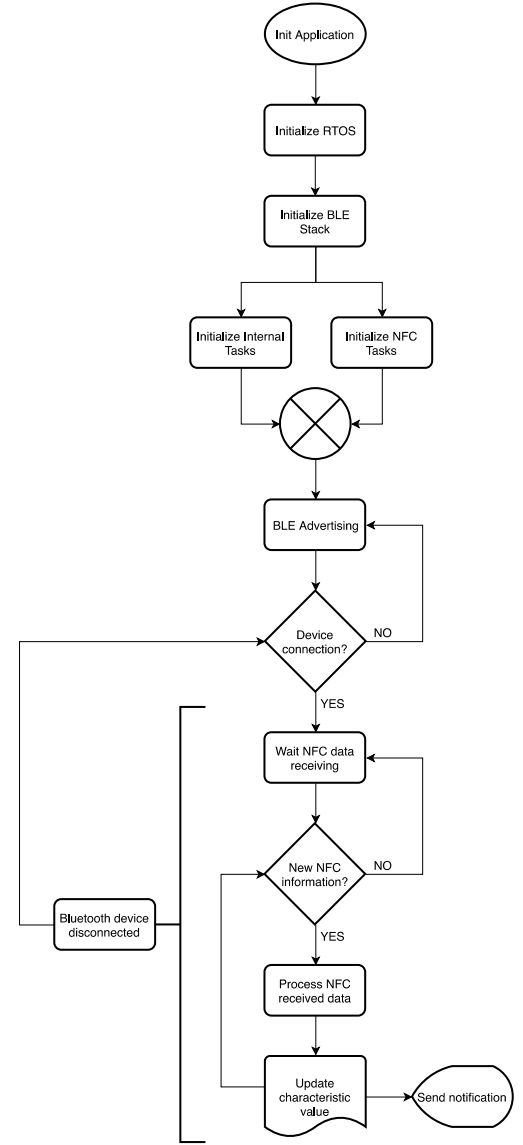

Fig. 8. Flowchart of the BLE stack.

useful when the user is asleep due to movements that are made involuntarily during sleep;

- Alarm for low battery level. The application alerts when the remaining range of the device is about 4 hours. Thus, the user can anticipate charging the battery of the device;

- Alarm by disconnection of the device. When the device loses the connection with the smartphone, to which it is linked, the application waits a period of parameter seconds waiting for a reconnection with the device. If, after that time, the communication has not been reestablished, the application will warn the user with an alarm, so that the user is aware that the system is not acting, and thus avoid trusting that it is being controlled. It also allows the possibility of making a warning call to another mobile phone notifying the situation;

- Cloud storage of the values. The user can create a MongoDB database with a certain structure. From this, configure the application, so that when the device makes a measurement on the sensor, store it in the online database as long as the smartphone has access to the Internet, either via $\mathrm{Wi}-\mathrm{Fi}$ or via mobile data.

\section{APPLICATIONS}

One of the applications, in which the device can be used, is to convert an NFC thermometer or temperature biopatch [9] in a continuous temperature control system. The device allows reading the stored values in the tag on demand without need to bring the reader closer and it sends the information to the BLE device, like a smartphone. In addition, it allows managing limits to launch alarms or to send a periodic notification with temperature values to 
another device. The values stored in the tag can also be periodically uploaded to a remote database. This allows accessing the information from anywhere. Figure 10 shows a graph with temperature evolution in a user's temperature biopatch.

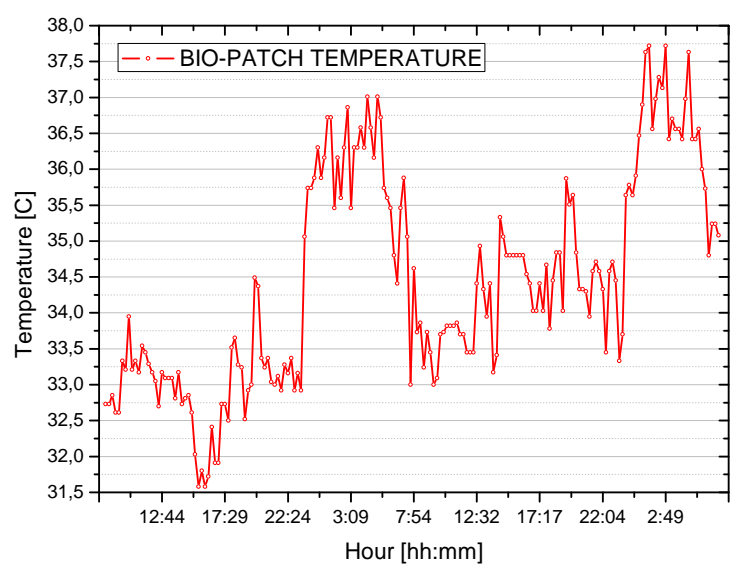

Fig. 10. Example of reading the temperature value of a wearable biopatch.

Besides, the proposed device has been used to read the glucose values of a commercial flash glucose-monitoring sensor [10]. This has allowed the monitoring of children and dependent people. Furthermore, it can be also used by blind and visually impaired users through a voice specific application as the app reads on demand the value of the glucose. Figure 12 shows a snapshot, including a view of the Nightscout [11] viewer that stores the values.
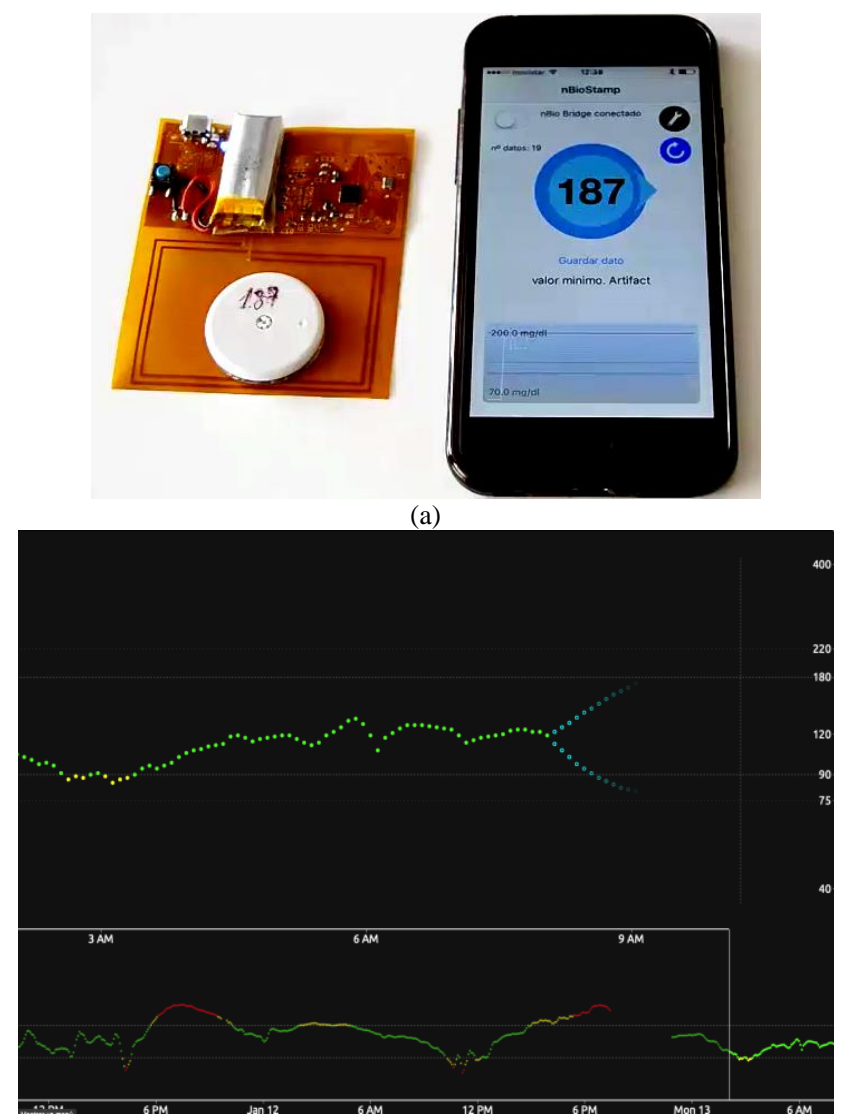

(b)

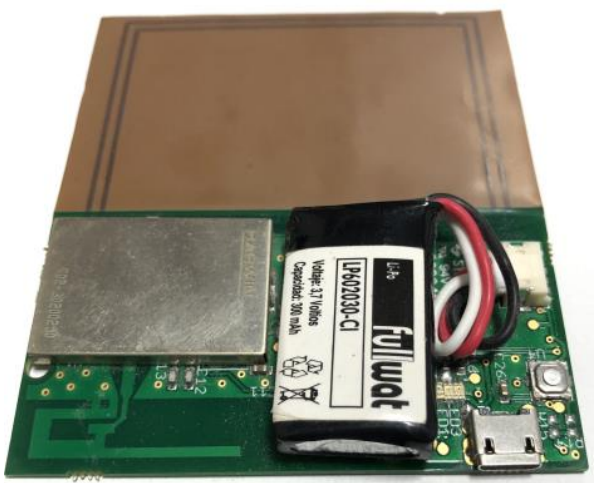

(c)

Fig. 11. (a) Example of reading the glucose value of a commercial flash sensor; (b) Nightscout view of uploaded values; (c) snapshot of the electronics.

\section{CONCLUSIONS}

A bridge of NFC-to-BLE allows implementing continuous monitoring from flash monitoring devices giving a high flexibility in many particular applications. For example, this system has been proven effective in daily use. 500 bridge units were manufactured for use with the noninvasive FreestyleLibre flash sensor, and they have been in use for over 18 months. Currently, version 2 of the FreestlyeLibre incorporates BLE so it no longer uses the bridge. The possibility of converting a flash sensor to a continuous sensor allows designing more efficient solutions in terms of energy consumption.

\section{CONFLICTS OF INTEREST}

The authors declare that they have no conflicts of interest.

\section{REFERENCES}

[1] Apple Watch Series 4, Apple. [Online]. Available: https://www.apple.com/es/apple-watch-series-4

[2] Embrace Seizure Detection, Empatica. [Online]. Available: https://www.empatica.com/embrace

[3] P. Brown, "The Future of Healthcare May Reside in Your Smart Clothes", Mouser Electronics, Medical Applications. [Online] Available: https://www.mouser.es/applications/healthcare-mayreside-in-smart-clothing

[4] Hexoskin Wearable Body Metrics, Hexoskin Smart Shirts - Cardiac, Respiratory, Sleep and Activity Metrics. [Online]. Available: https://www.hexoskin.com

[5] "FreeStyle Libre - Glucose Monitoring System - Diabetes Care", Abbot, Abbott Laboratories Limited, 2019. [Online]. Available: https://www.freestylelibre.co.uk/libre/

[6] “TempTraq, Wireless Baby Thermometer", Blue Spark Technologies, Inc., Blue Spark, 2020. [Online]. Available: https://www.temptraq.com/Home

[7] "femSense, Ovulation Detection", SteadySense GmbH, SteadySense 2020. [Online]. Available: https://www.femsense.com/en/

[8] K. Yamashita et al., "A $38 \mu \mathrm{A}$ wearable biosignal monitoring system with near field communication", in Proc. of 2013 IEEE 11th International New Circuits and Systems Conference (NEWCAS), Paris, 2013, pp. 1-4. DOI: 10.1109/NEWCAS.2013.6573637.

[9] J. M. Vicente, E. Avila-Navarro, C. G. Juan, N. García, and J. M. Sabater-Navarro, "Design of a wearable bio-patch for monitoring patient's temperature", in Proc. of 2016 38th Annual International Conference of the IEEE Engineering in Medicine and Biology Society $(E M B C)$, Orlando, FL, 2016, pp. 4792-4795. DOI: 10.1109/EMBC.2016.7591799.

[10] nBio Glucoguard. [Online]. Available: http://nbio.umh.es/glucoguard [11] Nightscout project. [Online]. Available: http://www.nightscout.info 